\title{
ANÁLISIS DE LA GESTIÓN DE PROYECTOS DE DESARROLLO CON UN MODELO DE POLÍTICAS PÚBLICAS. EL CASO DEL PROYECTO DESARROLLO SOCIAL INTEGRADO Y SOSTENIBLE (PRODESIS) EN CHIAPAS $^{*}$
}

\author{
Manuel Ignacio Martínez Espinoza \\ manuel.martinez.espinoza@gmail.com \\ Posdoctorado en Proimmse-IIA \\ Universidad Nacional Autónoma de México
}

\section{RESUMEN}

Los estudios de políticas públicas son una herramienta analítica provechosa para la gestión pública pues permiten examinar el diseño, proceso y resultados de una intervención pública evaluando el contexto en el que ésta se implementa. No obstante, las evaluaciones sobre los proyectos de la cooperación internacional al desarrollo prácticamente han ignorado los estudios de las políticas públicas. Es así que este trabajo expone una propuesta de modelo de análisis de la gestión de los proyectos de cooperación al desarrollo basada en el campo de las políticas públicas. Dicha propuesta se aplica en el análisis de la gestión pública de un proyecto de cooperación al desarrollo implementado en el estado de Chiapas: el Proyecto Desarrollo Social Integrado y Sostenible (Prodesis).

Palabras clave: cooperación al desarrollo, políticas públicas, Comisión Europea, Chiapas

\footnotetext{
* Este trabajo resume los resultados de un caso de estudio de la tesis doctoral "La participación de los pueblos indígenas en los proyectos de cooperación al desarrollo de la Comisión Europea, los casos de México y Guatemala”, elaborada por el autor. Véase Martínez Espinoza (2009).
} 


\begin{abstract}
Public policy studies are a useful analytic tool for public management since they make it possible to examine the design, process and results of a public intervention, evaluating the context in which it is being implemented. Nevertheless, the assessment of international development cooperation projects has practically ignored these studies. This work thus presents a model for analyzing the management of development cooperation projects based on the field of public policies. This proposal is used to analyze public management of a cooperation development project implemented in the State of Chiapas: the Integrated and Sustainable Social Development Project called Prodesis.
\end{abstract}

Key words: development cooperation, public policies, European Commission, Chiapas.

\footnotetext{
* This work summarizes the results of a case study in the doctoral thesis "Participation of indigenous peoples in European Commission development cooperation projects: the cases of Mexico and Guatemala", developed by the author. See Martínez Espinoza (2009).
} 


\section{INTRODUCCIÓN}

En el presente artículo se analiza la gestión pública del «Proyecto Desarrollo Social Integrado y Sostenible» (Prodesis), el cual fue cofinanciado por la Comisión Europea y el gobierno del estado de Chiapas e implementado en la entidad de 2004 a 2008. El análisis se realiza empleando una propuesta de modelo de evaluación de políticas públicas.

La evaluación es una fase fundamental en la gestión pública pues coadyuva a su eficiencia, eficacia y legitimidad mediante la retroalimentación objetiva de sus acciones. ${ }^{1}$ Aunque existen numerosas tipologías al respecto, ${ }^{2}$ la evaluación puede clasificarse en tres modelos generales: evaluación de diseño, del proceso y de resultados. ${ }^{3}$ La primera tiene como objetivo analizar la racionalidad y coherencia en el diseño de las medidas de gestión. La segunda consiste en examinar la forma en que se gestiona y ejecutan esas medidas. La tercera evalúa el alcance de los objetivos establecidos.

En el caso de la cooperación internacional para el desarrollo, ${ }^{4}$ se ha buscado que las intervenciones ${ }^{5}$ de los donantes tengan un cierto nivel de homogeneidad. Es así que, para beneficiarse de un lenguaje común para sus actuaciones, en la cooperación al desarrollo se ha adoptado un método de trabajo conocido como «gestión del ciclo de proyecto» (GCP). ${ }^{6}$ La GCP sustenta su desenvolvimiento en el enfoque del marco lógico (EML), el cual es una herramienta analítica para la planificación y gestión de proyectos orientados por objetivos que se aplica en la mayoría de organizaciones que gestionan proyectos de cooperación al desarrollo (entre ellas, la Unión Europea y el Banco Mundial). ${ }^{7}$

De esta manera, las evaluaciones en la cooperación internacional al desarrollo, si bien han adquirido diversas modalidades, ${ }^{8}$ han tomado como su eje estructural el EML, y particularmente los llamados criterios de evaluación del mismo (eficacia, eficiencia, pertinencia, impacto y viabilidad), por lo que, retomando la clasificación atrás asentada, han sido más evaluaciones de resultados o de diseño que de proceso. 
Es indudable que las condiciones sociales, económicas y, ante todo, políticas en el territorio de implementación influyen en el resultado del proyecto pues son variables que afectan su ejecución y los gestores no tienen un control directo sobre ellas. Resulta oportuno entonces examinar esas condiciones externas como una variable dependiente que repercute en los resultados del proyecto. Es así que un estudio de políticas públicas emerge como una opción analítica relevante para la evaluación de proyectos de cooperación al desarrollo. ${ }^{9}$

Aunque se ha extendido su comprensión de forma contraria, la gestión pública no debe ser una actividad monopólica de gobiernos sino un ejercicio facultativo de recursos instrumentales que, enmarcado en un cúmulo de valores democráticos, administra institucionalmente bienes y servicios en el espacio público. Desde este enfoque es factible concebir la cooperación internacional al desarrollo como un asunto de gestión pública.

Dado que el espacio de lo público es el contexto que orienta la gestación, formulación, implementación y evaluación de las políticas públicas, existe un nexo indisoluble, cercano a la tautología, entre éstas y la gestión pública (Uvalle 2002). Sin embargo, prácticamente son inexistentes las evaluaciones sobre proyectos de la cooperación internacional al desarrollo que sustenten sus herramientas analíticas en el campo de las políticas públicas.

Partiendo de estas premisas, el presente texto expone una propuesta de modelo de análisis de políticas públicas para la evaluación de los proyectos de cooperación al desarrollo. La propuesta se emplea para evaluar la gestión pública de una intervención al desarrollo implementada en la selva Lacandona de 2004 a 2008: el Prodesis.

Este trabajo se divide en cuatro apartados. En el primero se expone el modelo de análisis de políticas públicas. En el segundo se describen los planteamientos básicos del Prodesis. En el tercero se evalúa su gestión a partir del modelo de análisis de políticas públicas 
propuesto y, finalmente, en el cuarto apartado se exponen las conclusiones del artículo.

\section{EL MODELO DE ANÁlisiS DE POLÍTICAS PÚBLICAS PARA LA EVALUACIÓN DE LOS PROYECTOS DE COOPERACIÓN AL DESARROLLO}

\section{FUNDAMENTOS TEÓRICOS DEL MODELO}

Las políticas públicas, surgidas como disciplina en el mundo anglosajón, ${ }^{10}$ pueden definirse como «el conjunto de acciones, de procesos, de interacciones e intercambios entre actores que tienen lugar en los ámbitos del poder político» (Grau 2002: 34).

Reconociendo la porosidad de sus fronteras y la multiplicidad de sus denominaciones, ${ }^{11}$ en el presente artículo se entiende el estudio de las políticas públicas como la investigación interdisciplinaria, aunque con énfasis en la politología, que permite examinar, comprender y explicar la configuración, el diseño, el funcionamiento y las consecuencias de las medidas operativas de la gestión pública (Martínez Espinoza 2009).

Como asiente Aguilar (2007), en el estudio de las políticas existen dos posiciones: la noción «racional» y la noción «transaccional» de la política. ${ }^{12}$ Advirtiendo tales posturas, la propuesta de modelo de análisis enfatiza la noción «transaccional», esto es, el análisis del poder político o, lo que es lo mismo, quién obtiene qué, por qué, cuándo y las consecuencias de ello (Grau 2002: 34). Tal noción resulta conveniente cuando se admite que las políticas públicas son una arena donde, si bien converge lo multidisciplinario, lo determinante es el factor político: la intervención de actores, proyectos, procesos e instituciones políticas. El enfoque de este modelo de análisis se justifica además por el territorio donde operó el proyecto seleccionado: Chiapas, lugar donde predomina un estilo de hechura de políticas enraizado precisamente en la noción transaccional de la política (Burguete y Reyes 2002). El modelo emplea, entonces, una metodología de análisis acorde con el contexto en el que se inserta: el modelo autoritario en transición (Cabrero 2000). ${ }^{13}$ 
El modelo de evaluación se sustenta en el entendimiento de que, por un lado, la política pública objeto de estudio (los proyectos de cooperación) es resultado de acuerdos básicos entre los diferentes actores decisorios de los sistemas políticos y, por otro lado, esos acuerdos son consumados por una organización que trabaja en un territorio espacial y temático específico pero que participa en diversos subsistemas que incluyen cinco niveles para el caso que se analiza: internacional (europeo), nacional (mexicano), intermedio (estatal), local (municipal) y comunal. Reconociendo tales postulados, el modelo se enfoca en el estudio del desempeño de la organización gestora del proyecto y la interacción con su contexto en tres fases de la política pública. En el siguiente apartado se expone su metodología.

LA METODOLOGÍA DEL MODELO DE ANÁLISIS DE POLÍTICAS PÚBLICAS PARA LA EVALUACIÓN DE LOS PROYECTOS DE COOPERACIÓN AL DESARROLLO

Básicamente, el modelo de análisis propuesto se enfoca en el desempeño de la organización gestora del proyecto y la interacción con su contexto en tres fases de la política pública. Sus pilares son el esquema de fases de políticas públicas y las perspectivas de análisis basadas en el proceso organizacional, en los actores (pluralismo), y en las variables estructurales (neomarxismo) e institucionales (neoinstitucionalismo) (ver gráfico 1).

En primer lugar, el esquema de fases de las políticas públicas organiza su estudio concibiéndolas como intervenciones con procesos, si bien no lineales sí con una secuencia inicial y final. Aunque se reconocen las limitaciones de esta perspectiva (las políticas suelen contener procesos irregulares), el esquema de fases se utiliza para describir analíticamente el inicio, desarrollo y conclusión del proyecto a investigar (o, lo que es lo mismo, sus planteamientos, su aplicación y sus resultados). Siguiendo a Ballart (1993), en el modelo se plantea el estudio de tres fases: formulación, implementación y evaluación. ${ }^{14}$ 
En segundo lugar, la perspectiva del proceso organizacional emerge del reconocimiento de que «las políticas no se pueden comprender al margen y separadamente de los medios de su ejecución» (Elmore 2003: 185). El modelo analiza la organización gestora de los proyectos de desarrollo siguiendo los postulados de Elmore (2003), y Osuna y Márquez (2000).

Dada su afectación y participación, los actores son un elemento esencial en las intervenciones al desarrollo. En virtud de ello, y bajo las premisas de Meltsner (2007), Pressman y Wildavsky (1998), Rein y Rabinovitz (2003) y Vedung (1996), el modelo identifica y clasifica a los actores que intervienen en los proyectos de cooperación.

En cuarto lugar, dada su relevancia para el curso de una política pública, el modelo también asume las condiciones externas a la intervención como un factor de análisis. Se retoma así una perspectiva estructural en la cual, considerando los enfoques de Sabatier y Mazmanian (2003), Osuna y Márquez (2000), van Meter y van Horn (2003) y del EML, se valoran las condiciones sociales, políticas y económicas del territorio donde la política se implementa.

Finalmente, el modelo de análisis también reconoce la importancia de las instituciones para el diseño, ejecución y evaluación de las políticas públicas. Siguiendo a North (1995), March y Olsen (1997) y Peters (2003), una institución política se define como el marco (formal o informal) que determina las fronteras de los procesos políticos dando estabilidad y certidumbre a la interacción de los actores. Son, como lo asienta Sánchez Ferrer (2002), las normas, valores y reglas de lo político. ${ }^{15}$

El Gráfico 1 expone esquemáticamente el modelo de análisis propuesto. 
Gráfico 1: Modelo de análisis de políticas públicas para la evaluación de los proyectos de cooperación al desarrollo

\section{Bases:}

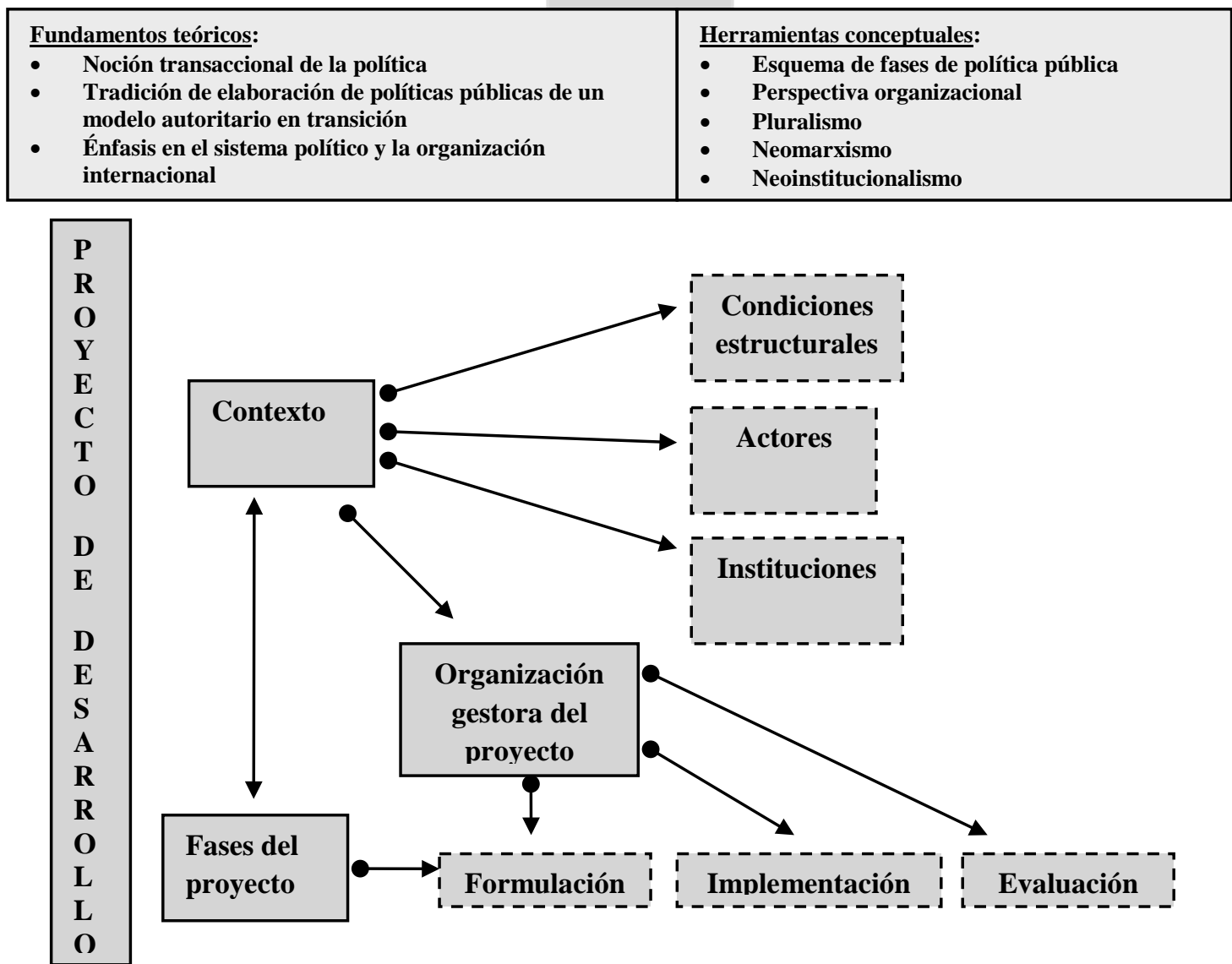

Fuente: Elaboración propia.

\section{LIMITACIONES DEL MODELO DE ANÁLISIS PROPUESTO}

El modelo expuesto plantea una ruta analítica para una evaluación de los proyectos de cooperación que, desde el campo de las políticas, integre los tres tipos de evaluaciones generales (diseño, proceso y resultados); por lo tanto, aunque se pruebe su utilidad, no se postula como un modelo acabado de evaluación pues no está exento de limitantes.

En primer lugar, la aproximación metodológica tiene principalmente un carácter cualitativo. 
Es decir, que sin ausencia total de información cuantitativa, el modelo se basa más en técnicas vinculadas a la interpretación de la realidad que en técnicas vinculadas a la interpretación de datos duros. ${ }^{16}$ Se reconoce entonces que el énfasis en la investigación cualitativa conlleva un importante riesgo de validez y posibilidades de sesgo. Para controlar tal riesgo, amén de la observación participante, se realizaron entrevistas a todos los actores vinculados al campo temático del proyecto usando la misma batería de preguntas. Así, la triangulación de fuentes se postuló como garantía de validez en la aplicación del modelo.

Por su parte, el modelo también se concentra en lo que Aguilar (2007) catalogó como «noción transaccional de la política». Ante tal énfasis, los enfoques técnicos, administrativos y económicos de análisis de políticas son considerados como complementarios al enfoque político. ${ }^{17}$

Finalmente, en tercer lugar, dado que el modelo de análisis estudia el desempeño de la organización gestora del proyecto y la interacción con su contexto en tres fases de la política pública, se concentra en el territorio espacial y temático de la implementación. Por lo anterior, se excluye el análisis de la estructura de la Comisión Europea y de la política europea de cooperación en general (es decir, un enfoque de relaciones internacionales), pues tal enfoque puede ampliar temporal y temáticamente el estudio.

No obstante, aun con las limitaciones señaladas el modelo es confiable para la evaluación de la gestión pública de los proyectos de cooperación debido a tres propiedades:

1) Reduce los sesgos de confirmación y disconformidad dando validez a la recopilación y análisis de la información pues evita buscarla o interpretarla unidireccionalmente.

2) Impide la deformación profesional; o sea, que coadyuva para que el análisis del fenómeno vaya más allá del prisma de la politología. De esta forma, es factible incluir 
postulados de otros campos del conocimiento para un análisis interdisciplinario desde la ciencia política.

3) Cuenta con un pilar del método científico: la reproducibilidad. Es decir, que el método puede repetirse para volver a comprobar los resultados de un estudio de forma empírica.

En los apartados subsiguientes se aplica el modelo abordando como caso de estudio un proyecto de cooperación implementado en Chiapas: el Prodesis, del cual se exponen a continuación sus características generales.

\section{CARACTERÍSTICAS GENERALES DEL PRODESIS}

El Prodesis fue un proyecto de cooperación al desarrollo cofinanciado por la Comisión Europea y el gobierno del estado de Chiapas; este último, fue también el responsable de su implementación en la selva Lacandona.

Originalmente, el proyecto fue planteado para cuatro años, de 2004 a 2007, en dos etapas: la fase ejecutiva (de 42 meses) y la de cierre (de 6 meses). Sin embargo, debido a retrasos en el inicio de sus operaciones, se firmó una ampliación de la fase ejecutiva, de manera que el proyecto concluyó en abril de 2008. El presupuesto del Prodesis se estableció en 31,000,000 euros, de los cuales 16,000,000 serían administrados por el gobierno del estado de Chiapas, y 15,000,000 por la Comisión Europea.

Según estimaciones de su organización gestora, el área de acción del Prodesis abarcaría una superficie de 1,258,157 hectáreas (equivalente al 16\% del territorio de Chiapas). La población beneficiaria del proyecto se estimó en 155,000 habitantes de 830 localidades, las cuales se agruparían en 16 microrregiones de siete municipios del estado: Ocosingo, Las Margaritas, Maravilla Tenejapa, Marqués de Comillas, Benemérito de las Américas, La Trinitaria y La Independencia ${ }^{18}$ (Prodesis 2004: 14). 
A partir de su diagnóstico de la selva Lacandona, el Prodesis se planteó tres objetivos generales: 1) la reducción de la pobreza en la zona; 2) la disminución de la presión sobre los recursos naturales; y 3) la reformulación de las políticas de desarrollo social en un sentido de desarrollo territorial, participativo y sustentable (Prodesis 2004: 7).

El Prodesis diseñó tres acciones para cumplir con sus objetivos: aplicar nuevas prácticas de planeación participativa, difundir la viabilidad de sistemas innovadores de producción sustentable y proporcionar a los actores un servicio de información oportuna.

Una de las metas más importantes del Prodesis fue la de promover la creación de «espacios de facilitación», es decir, espacios donde los ejecutores locales (comunidades, organizaciones), los municipios, las instituciones gubernamentales y las instituciones de apoyo (organizaciones de la sociedad civil, centros de investigación) lograran concertar acuerdos para fomentar acciones coordinadas de desarrollo local. Es así que los llamados consejos microrregionales (en adelante, CMR) fueron una apuesta fundamental del Prodesis en términos de participación, reorganización política y apropiación social. Se definió así que habría un consejo microrregional en cada microrregión.

La sede central del proyecto se estableció en la ciudad de Tuxtla Gutiérrez y se crearon tres coordinaciones regionales para atender a las 16 microrregiones. Esas subsedes ubicaron sus cabeceras en Palenque, Ocosingo y Comitán.

Los planteamientos del Prodesis también incluyeron ejes estratégicos transversales (segmentos temáticos de interés para el proyecto). Estos fueron ${ }^{19}$ :

a) Participación social: ampliar la participación y representatividad de las comunidades.

b) Equidad de género: impulsar, fomentar y fortalecer con equidad la participación y el acceso de las mujeres a las acciones del proyecto. 
c) Derechos de los pueblos indígenas: promover la participación de los indígenas en la definición de sus propios procesos de desarrollo sustentable conforme al Convenio 169 de la Organización Internacional del Trabajo.

d) Aprovechamiento sustentable de los recursos naturales: establecer prácticas productivas sustentables.

e) Información y transparencia en la gestión y en la rendición de cuentas.

Finalmente, en cuanto a su estructura organizativa, el Prodesis se constituyó partiendo del sector de la política de desarrollo social del beneficiario (la Secretaría de Desarrollo Social del gobierno del estado de Chiapas), estableciendo la responsabilidad de ejecución del proyecto en una unidad gestora autónoma (la unidad de coordinación), con la participación técnica de la representación de la Comisión Europea (la asistencia técnica internacional) y con el apoyo de un consejo asesor (el comité consultivo), estructurando sus acciones en los tres componentes básicos del proyecto (planeación, producción sustentable, y el observatorio) en un territorio dividido en 16 microrregiones las cuales, a su vez, se coordinan en tres regiones (Palenque, Ocosingo, Comitán) (ver Gráfico 2). 
Gráfico 2. Organigrama de la estructura institucional del Prodesis

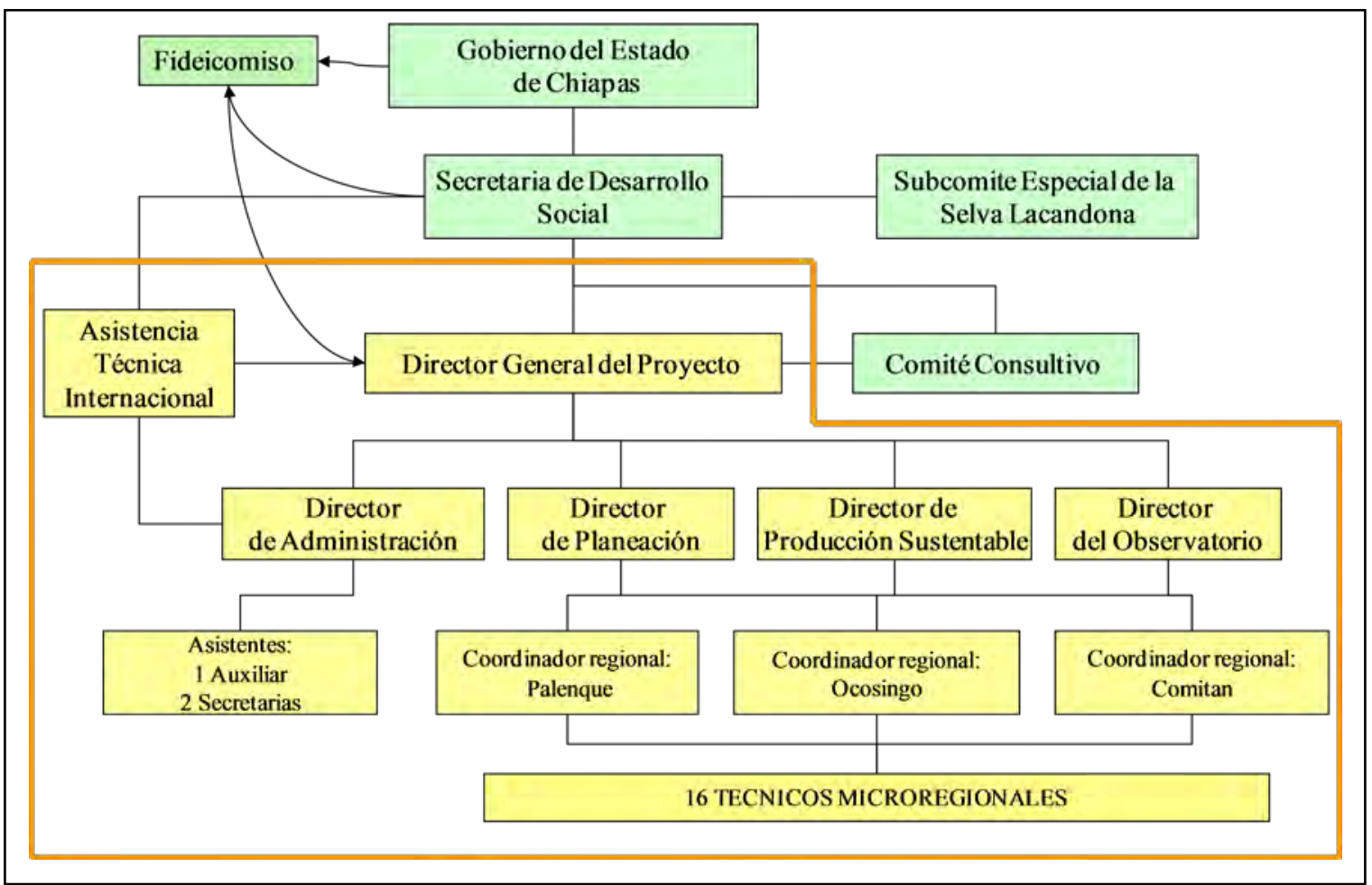

Fuente:http://www.Prodesis.chiapas.gob.mx/?\%BFQu\%E9_es_el_PRODESIS\%3F/Estructura_institucional_d el_proyecto [Consulta: 11 de enero de 2008].

De esta forma, el Prodesis comenzó a operar a principios de 2004 concluyendo en abril de 2008. En los siguientes apartados se evalúa su gestión empleando el modelo de análisis de políticas públicas propuesto.

\section{ANÁLISIS DE LA GESTIÓN DEL PRODESIS CON EL MODELO DE POLÍTICAS PÚBLICAS ${ }^{20}$}

\section{ESPACIO DE LA POLÍTICA Y AGENDA DEL PRODESIS}

La selva Lacandona es uno de los macizos forestales más importantes de Mesoamérica en términos de diversidad biológica y de regulación climática e hídrica. Con su casi medio millón de habitantes, ${ }^{21}$ es un territorio con múltiples, complejos e interrelacionados problemas sociopolíticos que, sumados al deterioro ambiental y su característica de frontera 
internacional, convierten a la región en una zona de atención especial y urgente.

Evidentemente, las políticas del Estado mexicano en la región (o la ausencia de éstas) han influido decisivamente en cuestiones como el aprovechamiento de los recursos naturales, la distribución de la tierra, el asentamiento de comunidades y la geografía política. ${ }^{22}$ En síntesis, las políticas de desarrollo social en Chiapas han tenido tres características: han sido ineficaces para combatir la marginación de la población, se han asentado en enfoques paternalistas y, una buena parte de ellas, se han orientado al control político de los beneficiarios (Camacho y Lomelí 2000). Ese tipo de políticas ha contribuido a generar una cultura política clientelar y un clima de desconfianza entre los colonos de la Lacandona.

En términos de proyectos de desarrollo en la selva, sobresalen el programa Cañadas (en adelante, PC) y el Programa Integral para el Desarrollo Sustentable de la Selva (PIDSS).

El PC, implementado por la Secretaría de Desarrollo Social del gobierno federal y acompañado por el Ejército, es uno de los programas más polémicos que han operado en la región. Surgido en el marco del levantamiento zapatista, el PC contó con un presupuesto oneroso que utilizó para crear infraestructura de comunicaciones y otorgar apoyos materiales a las comunidades y organizaciones opuestas al zapatismo, por lo que el programa fue calificado como «proyecto contrainsurgente» (Muench 2008 y entrevistas de campo). El PC fue cancelado en el año 2000.

Anunciado poco después de la alternancia gubernamental federal y estatal del año 2000, el PIDSS sustituyó al PC como programa de desarrollo en la selva por lo que, en el marco de reacomodos entre los actores políticos, significó un proceso de deshegemonización de los grupos empoderados por el PC mediante alianzas con organizaciones afines al nuevo gobierno $^{23}$ (entrevistas de campo). Fue en ese contexto cuando en 2003 la Comisión Europea, aprovechando la inercia del PIDSS, seleccionó la selva Lacandona como el 
territorio para financiar un proyecto de desarrollo en México, surgiendo así el Prodesis.

Como se puede inferir, el desarrollo sustentable, el ordenamiento territorial y participativo y la degradación ambiental de la Lacandona (objetivos del Prodesis) han integrado la agenda sistémica del Estado mexicano pues su atención ha estado definida por la mitigación de aspectos puntuales (control de empresas forestales, aprovechamiento de recursos naturales, levantamiento zapatista), siendo entonces meramente coyuntural. ${ }^{24}$

A su vez, la implementación de políticas en la selva se ha caracterizado por el bajo nivel de consenso y negociación con los actores, siendo más bien prácticas sustentadas en el clientelismo político y la coerción. Por ello, las políticas en la Lacandona han tenido un estilo más reactivo e impositivo que anticipativo y consensual. ${ }^{25} \mathrm{El}$ espacio de la política del Prodesis, ${ }^{26}$ entonces, resultaba altamente complejo en términos económicos, extensamente segmentado en términos sociales, y hondamente conflictivo en términos políticos.

De esta manera, surgió el Prodesis como una nueva oportunidad para generar prácticas efectivas de combate a la pobreza, ordenamiento territorial, participación y aprovechamiento sustentable de los recursos de la selva. En los siguientes apartados se analiza su diseño, su organización gestora, su implementación y los resultados alcanzados.

\section{FORMULACIÓN Y DISEÑO DEL PRODESIS}

La formulación del Prodesis se enfrentó a dos obstáculos: la coordinación de las reglas operativas entre el cooperante y el beneficiario (lo que ocasionó la redacción de hasta tres programas operativos globales -POG- distintos) y la instalación del instrumento financiero para la administración de los recursos, útil para equiparar la divisa europea con la mexicana. Esto retrasó el inicio de operaciones del proyecto y, como se verá más adelante, afectó la implementación del mismo. 
Con respecto al diseño del Prodesis, la identificación de los problemas y su propuesta de intervención resultó congruente debido a la coherencia entre el árbol de problemas y de intervenciones.

Sin embargo, un análisis más detallado del POG del Prodesis evidencia que la identificación de problemas y actores en la selva resultó insuficiente pues algunos se omitieron o fueron abordados de forma superficial. Tal es el caso del clientelismo, los tráficos ilegales y la breve alusión al Ejército Zapatista de Liberación Nacional. ${ }^{27}$ En este aspecto, llama la atención que el POG del Prodesis identificó cerca de 70 actores en la Lacandona (Prodesis 2004: 18-20) pero no incluyó a partidos políticos, Iglesias, instituciones académicas ni otros proyectos de la cooperación internacional. Más aún, el Prodesis pronosticó que las organizaciones identificadas en la selva, así como las instituciones gubernamentales, apoyarían el proyecto. Sin embargo, en la práctica, si bien el Prodesis contó con un sólido apoyo de actores puntuales (grupos beneficiados por el programa), en la práctica tuvo que enfrentarse a una inestabilidad de posturas y a férreos rechazos. Por ello, algunos consultores lo calificaron como un proyecto con «diseño pertinente pero no viable» (Di Giacinto et al. 2007).

Como producto de su formulación y diseño, se constituyó la organización gestora del Prodesis. Las características de dicha organización se analizan en el siguiente apartado.

\section{LA ORGANIZACIÓN GESTORA DEL PRODESIS}

La estructura gestora del Prodesis se constituyó con personal nacional y de la asistencia técnica internacional, además de una figura de retroalimentación nombrada comité consultivo, por lo que su diseño buscó incorporar elementos de contrapeso para el equilibrio de las decisiones. No obstante, la estructura del Prodesis encaró diversos inconvenientes que minaron su accionar, tales como sustituciones en su plantilla (todo el 
personal, incluidos los directores, fueron sustituidos en alguna ocasión) y conflictos entre la asistencia técnica internacional, la dirección general del proyecto y el comité consultivo. De hecho, este último fue un espacio muy crítico hacia la dirección del proyecto, llegando al grado de que muchas organizaciones y personalidades que originalmente lo integraron, tiempo después lo abandonaron. De esta manera, los conflictos en sus estructuras afectaron la eficiencia del Prodesis (Di Giacinto et al. 2007).

Por su parte, también la administración del presupuesto fue un asunto problemático, siendo éste el aspecto que más se le impugnó a la organización gestora: muchos de los miembros de las microrregiones criticaron la administración de los recursos en el Prodesis aduciendo ya sea escasez, ineficiencia, corrupción, retrasos y arbitrariedad en la asignación, o excesivo financiamiento en estudios y consultorías poco útiles. A esto hay que agregar los diversos problemas que se presentaron para establecer las cuentas de operación del proyecto.

En resumen, la inestabilidad en el organigrama del Prodesis y los problemas en el ejercicio de los recursos afectaron el desempeño de la organización gestora del Prodesis, minando en gran parte la implementación del mismo, como se verá en el siguiente apartado.

\section{LA IMPLEMENTACIÓN DEL PRODESIS}

El Prodesis inició en 2004. Debido a las dilaciones en la fase de formulación, sólo se ejecutó menos del 6\% del presupuesto programado para ese año (99,000 euros de $1,700,000)$.

Ante ello, el programa operativo del 2005 incorporó actividades no implementadas en el año previo. Aún así, en ese año también hubo retrasos administrativos, por lo que su cumplimiento presupuestario fue, contando los dos años de operación, de 7,500,000 euros (equivalente a sólo el 57\% de lo programado originalmente). Es así como, a partir de un 
informe de sostenibilidad, la Comisión Europea aprobó en 2005 una extensión del Prodesis por diez meses más, estableciendo el cierre técnico operativo del proyecto para abril del 2008, más un periodo de seis meses para el cierre administrativo financiero.

En el año 2006, aunque siguió habiendo demoras (el programa operativo anual 3, para un periodo de 18 meses correspondientes a 2006 y 2007, se aceptó tardíamente), se aceleraron las operaciones administrativas. En los tres años de operación del proyecto (2004, 2005 y 2006), se ejerció la cantidad de 20,447,000 euros, lo que correspondió a sólo el 66\% del presupuesto previsto originalmente.

Durante 2007 y el primer cuatrimestre de 2008 se realizó la fase final de ejecución. En ese periodo se realizaron las acciones que sufrieron retrasos en los años anteriores. De esta forma, en el segundo cuatrimestre de 2008 concluyó la fase de implementación del Prodesis, con un resultado de implementación discordante en cada uno de los componentes del proyecto (Muench 2008 y entrevistas de campo).

Es así como la implementación del proyecto se dilató por retrasos administrativos (sobre todo en la disposición de recursos y aprobación del POG y los distintos programas operativos), menoscabada por el desigual trabajo de los componentes y demeritada por la falta de interés y participación efectiva de distintas instituciones públicas. ${ }^{28}$

La implementación del Prodesis, entonces, estuvo condicionada por los tres imperativos identificados por Rein y Rabinovitz (2003: 148-149): el imperativo legal (las distintas disposiciones reglamentarias), el imperativo racional burocrático (la adecuación de los procesos según la racionalidad de las operaciones administrativas) y el imperativo consensual (la aceptación del proceso por parte de los actores participantes), resultando así un proceso titubeante e insuficiente con respecto a la ruta de implementación original. 
Ese tipo de implementación puede explicarse a través del esquema analítico propuesto por Pressman y Wildavsky (1998), en el sentido de que en la operación del Prodesis existieron «puntos de decisión» (actos de acuerdo para el desarrollo del programa) y «puntos claros» (aprobación de los participantes por separado), más decisivos que numerosos, los que, a su vez, demoraron considerablemente el desarrollo del proyecto afectando sus resultados, tal como se analizará en el siguiente apartado sobre las evaluaciones al Prodesis.

\section{LA EVALUACIÓN DEL PRODESIS}

El Prodesis fue evaluado externamente en tres ocasiones. La primera evaluación la realizó la Universidad Autónoma Metropolitana, quien primero valoró el desempeño del PIDSS y después el del Prodesis del año 2002 al 2007 (UAM 2003-2008).

Un segundo ejercicio evaluativo del Prodesis lo realizó el Instituto de Tecnología Social, una empresa consultora contratada por el propio proyecto. Esta evaluación se efectuó en 2007 y tuvo un carácter cuantitativo tomando como universo de estudio a las16 microrregiones y sobre la base de entrevistas a participantes en el proyecto. (TECSO 2007).

La tercera evaluación fue realizada por otra empresa consultora también en 2007, IBF en consorcio con B.A.a. Consultors SL. Se caracterizó como misión de evaluación de medio término y se enfocó en los aspectos de sostenibilidad de los resultados del proyecto, evaluando la pertinencia, eficacia, eficiencia, impacto, sostenibilidad y coherencia interna.

Un análisis de los ejercicios evaluativos señalados permite encontrar semejanzas en sus resultados. Así, las tres evaluaciones coinciden en concluir que los principales impactos de carácter beneficioso del Prodesis fueron el incremento en número de la participación de los colonos de la selva en su CMR, la capacitación de algunos miembros de las comunidades en temas de producción y derechos humanos, el equipamiento de centros comunitarios, el aumento del ingreso autogenerado para algunos participantes, algunas prácticas de manejo 
sustentable de los recursos, así como una propuesta de ordenamiento territorial y un sistema de información potencialmente útil para el desarrollo sustentable.

Por su parte, las tres evaluaciones también coinciden en concluir que, en términos perjudiciales, el Prodesis tuvo efectos nulos en la consecución efectiva de sus objetivos trazados, la coordinación interinstitucional, el fortalecimiento institucional de los CMR, la equidad de género y étnica, la pacificación del conflicto político militar y la erradicación de una cultura política paternalista y clientelar en las comunidades de la selva. Además, añaden, el Prodesis incidió negativamente en la región mediante diversas acciones como la asignación tardía y discrecional de recursos, la contratación de algunas consultorías que, sin conocimiento del lugar y mediante trabajo poco consistente en la región, causaron problemas de coordinación en el proyecto, la imposición de proyectos productivos (algunos no sustentables), así como actos de corrupción y manipulación política; todos ellos, aspectos que agravaron la división en las comunidades y alentaron entre las organizaciones de la sociedad civil las sospechas de biopiratería bajo la efigie del Prodesis.

Es así como, debido a fallas en su diseño, estructura operativa, administración de recursos y concertación sociopolítica en su formulación e implementación, el Prodesis no sólo incumplió sus objetivos generales, sino que además desatendió sus ejes estratégicos transversales dando como resultado un proyecto de desarrollo ineficiente y severamente cuestionado en gran parte de las microrregiones donde se implementó.

\section{CONCLUSIONES}

Resulta una obviedad afirmar que Chiapas, y en particular la selva Lacandona, requieren políticas públicas y proyectos de cooperación al desarrollo social. El espacio de la política (policy space) se caracteriza por la pobreza extrema, la marginación profunda y el uso depredador de los recursos naturales. Junto a ello, el sistema político es de bajo nivel institucional, con estructuras oligárquicas y una cultura política basada en el mecenazgo y 
la violencia. Además, las instituciones políticas y las organizaciones sociales son abundantes y contrapuestas, lo que convierte al ejercicio político en una actividad de suma cero.

Asimismo, la región se ha caracterizado por intervenciones puntuales, coyunturales e impositivas que no sólo han sido ineficientes para el desarrollo social sino que inclusive han causado efectos contrarios, profundizando así conflictos territoriales, intracomunitarios y medioambientales. El PC fue, sin duda, el más claro ejemplo de un proyecto proclamado como de desarrollo social pero con objetivos, enfoques y resultados antagónicos al mismo.

No obstante, en los albores del nuevo milenio la situación dio un giro importante con la alternancia en los gobiernos federal y estatal, la cancelación del PC y el llamado para una nueva estrategia de desarrollo en la Lacandona. Poco después, surgió el interés de la Unión Europea por financiar un proyecto de cooperación en México. En 2003 se seleccionó Chiapas y se firmó el convenio de financiación para el Prodesis. Las prácticas de desarrollo social sustentable y participativo tendrían una nueva oportunidad en Chiapas.

Sin embargo, luego del análisis con el modelo de políticas públicas propuesto, es factible concluir que el Prodesis fue una oportunidad perdida para implementar políticas públicas efectivas, sustentables y legítimas para la Lacandona: de la amplia expectativa generada por los discursos de sustentabilidad, el apoyo institucional de la Unión Europea y la abundante suma de recursos económicos con que el Prodesis contó, quedaron sólo algunos proyectos sustentables, capacitación, algo de infraestructura, planes de desarrollo territorial y un importante sistema de información. Sin duda no es poco, pero a ello hay que sumarle el incumplimiento de sus tres objetivos generales y sus fracasos en la coordinación interinstitucional, en el fortalecimiento de los CMR, en la equidad de género y en la equidad étnica, así como las acusaciones de las comunidades participantes por la asignación tardía y desviada de los recursos, el trabajo deficiente de las consultorías, la imposición de 
proyectos y algunos actos de corrupción. El resultado es, como se pudo constatar en el trabajo de campo, decepcionante para quienes fueron el público objetivo del Prodesis.

Evidentemente, los factores causales de esos resultados defectuosos son diversos, y no todos atribuibles al Prodesis. No obstante, como el modelo de políticas públicas pudo mostrar, el proyecto tuvo fallos en sus fases como política pública, tales como la insuficiente identificación de actores y problemas, los retrasos administrativos, el cumplimiento del cronograma de trabajo, la asignación de recursos, la coordinación interinstitucional en el territorio y la permisividad de trabajos deficientes de las consultorías.

Al momento de la redacción de este artículo, se está evaluando el reinicio del Prodesis en otra región de Chiapas. Por lo tanto, es posible que para los gestores el Prodesis no fuese una posibilidad perdida, sino un proyecto de prueba, de manera que les sería de utilidad tomar nota de aquéllo que permite que una oportunidad se convierta en una experiencia rescatable. Del análisis del Prodesis con el modelo de políticas públicas propuesto se pueden extraer cinco recomendaciones a tener en cuenta para mejorar la gestión de las intervenciones de desarrollo en la selva Lacandona:

1) Considerar las estructuras políticas como enclaves determinantes para una intervención de desarrollo. En concreto, tomar en cuenta que la cultura política clientelar erosiona cualquier política pública planteada con enfoques de sustentabilidad y participación. Por lo tanto, podría ser de utilidad acompañar las intervenciones al desarrollo con actividades de educación cívica para la democracia que hagan énfasis en las competencias cívicas. ${ }^{29}$

2) Buscar contar con el apoyo efectivo de los tres órdenes de gobierno, así como de las instituciones públicas y gubernamentales que converjan en el territorio de implementación del proyecto. En este punto, el respaldo de la Comisión Europea es vital para generar 
inercias que no sólo favorezcan al proyecto en sí mismo, sino que hagan factible la anhelada coordinación interinstitucional.

3) Establecer mecanismos efectivos para el control y vigilancia tanto de las consultorías como de la administración de los recursos que, además de transparentar la ejecución del proyecto, legitimen su accionar entre la población. Esto puede traducirse en la creación de un comité de control de los recursos compuesto por ciudadanos, institutos de investigación, instituciones públicas y organizaciones sociales.

4) Reconsiderar las funciones y potestades del comité consultivo para convertirlo en un instrumento eficaz de retroalimentación del proyecto.

5) Realizar un análisis más profundo sobre la complejidad de problemas y actores que radican en la selva Lacandona de manera que el diagnóstico y las estrategias tengan un mayor nivel de pertinencia.

Aún en su gran diversidad, las evaluaciones de los proyectos de cooperación al desarrollo se han concentrado en tres aspectos puntuales: diseño, proceso de gestión y resultados. Ello ha permitido su especialización pero ha afectado su integralidad: generalmente, los tres aspectos han sido analizados de forma desvinculada medrando así el entendimiento holístico de las intervenciones al desarrollo.

A pesar de que su aplicación al Prodesis fue muy sintética por asuntos de espacio, ${ }^{30}$ el modelo de políticas públicas para la evaluación de los proyectos de cooperación al desarrollo que se presenta en este artículo resultó de utilidad en la medida que sirvió como un esquema que integró el diseño, el proceso y los resultados de un proyecto de desarrollo concentrándose en la organización gestora y su desempeño en el contexto de implementación. Con el modelo propuesto no se profundiza al máximo en cada uno de los 
aspectos pero se obtiene una panorámica integral que permite descifrar las fortalezas y debilidades del proyecto desde la mira de la organización gestora, su metodología y el contexto en el que se inserta.

El modelo de políticas públicas propuesto es, entonces, un aporte a considerar en la evaluación de los proyectos de cooperación en la medida que traslada su análisis al ámbito de la gestión pública, ese espacio donde poco se ha hecho para abordar estas intervenciones al desarrollo que, por definición, son de carácter público y deben ser evaluadas como tales. 


\section{BIBLIOGRAFÍA}

Aguilar Villanueva, Luis F., (editor), 2007, La hechura de las políticas públicas, Miguel Ángel Porrúa, $2^{a}$ reimpresión, México.

Alonso, José Antonio y Valpy Fitzgerald (coordinadores), 2003, Financiación del desarrollo y coherencia en las políticas de los donantes, Catarata, Madrid.

Arellano Gault, David, 1999, «De la administración pública a la nueva gestión: cinco dilemas», en Revista Conmemorativa del Colegio. El estado del arte de la administración pública, Colegio Nacional de Ciencias Políticas y Administración Pública, México, pp. 3547.

Ballart, Xavier, 1993, «Evaluación de políticas. Marco conceptual y organización institucional», en Revista de Estudios Políticos (Nueva Época), n. 80, abril-junio, CEPC, Madrid, pp. 199-224.

—, 1997, «Gestión pública, análisis y evaluación de políticas. Delimitación de contenidos y bibliografía», en Revista de Estudios Políticos (Nueva Época), n. 97, julioseptiembre, CEPC, Madrid, pp. 223-247.

Beltrán Villalva, Miguel, 2000, La acción pública en el régimen democrático, CEPC, Madrid.

Burguete, Araceli y María Eugenia Reyes (coordinadoras), 2002, La política social en Chiapas, Universidad de Ciencias y Artes de Chiapas, Tuxtla Gutiérrez.

Cabrero Mendoza, Enrique, 2000, «Usos y costumbres en la hechura de las políticas públicas en México. Límites de las Policy Sciences en contextos cultural y políticamente 
diferentes», en Gestión y política pública. Segundo semestre, v. IX, n. 002, CIDE, México, pp. 189-229.

—, 2000b, «Estudio introductorio», en La gestión pública: su situación actual, coordinado por Bozeman, Barry, Fondo de Cultura Económica, México, pp. 37-42.

Camacho, Dolores y Arturo Lomelí, 2002, «Consideraciones sobre la política social, la inversión pública y las organizaciones sociales en Chiapas», en La política social en Chiapas, coordinado por Reyes, María Eugenia y Araceli Burguete, Universidad de Ciencias y Artes de Chiapas, Tuxtla Gutiérrez.

De Vos, Jan, 2002, Una tierra para sembrar sueños. Historia reciente de la Selva Lacandona 1950-2000, CIESAS, FCE, México.

Di Giacinto, Piero, Jan Karremans y Thomas Pijnemburg, 2007, Evaluation of Project Integrated and Sustainable Social Development, Chiapas, México (PRODESIS). Ec Refrence n. ALA/B7-310/2003/5756, draft final report, IBF International Consulting \& B.A.a. Consultors, México.

Elder, Charles y Roger Cobb, 1996, «Formación de la agenda. El caso de la política de los ancianos», en Problemas públicos y agenda de gobierno, editado por Aguilar Villanueva, Luís, Miguel Ángel Porrúa, México, pp. 77-104.

Elmore, Richard F., 2003, «Modelos organizacionales para el análisis de la implementación de programas sociales», en La implementación de las políticas públicas, editado por Aguilar Villanueva, Luís, Miguel Ángel Porrúa, México, pp. 185-280.

Gómez Galán, Manuel y José Antonio Sanahuja (coordinadores), 2001, La cooperación al 
desarrollo en un mundo en cambio. Perspectivas sobre nuevos ámbitos de intervención, CIDEAL, Madrid.

Grau Creus, Mireia, 2002, «El estudio de las políticas públicas: enfoques y metodologías de análisis», en Análisis de políticas públicas en España: enfoques y casos, editado por Grau, Mireia y Araceli Mateos, Tirant lo Blanch, Valencia, pp. 29-58.

Guerrero, Omar, 2001, «Nuevos modelos de gestión pública», en Revista Digital Universitaria, v. 2, n. $3, \quad 30$ de septiembre, UNAM, México, en http://www.revista.unam.mx/vol.2/num3/art3/index.html. [Consulta: 2 de agosto de 2008].

Instituto Federal Electoral, 2003, Educar para la democracia, Documento de trabajo, Instituto Federal Electoral, México.

Iglesia-Caruncho, Manuel, 2005, El impacto económico y social de la cooperación para el desarrollo, Catarata, Instituto Universitario de Desarrollo y Cooperación, Madrid.

Instituto en Tecnología Social, AC, 2007, Evaluación inicial del programa, Proyecto Desarrollo Social Integrado y Sostenible, Instituto en Tecnología Social, Tuxtla Gutiérrez.

Instituto Nacional de Estadística, Geografía e Informática, 2005, Censo de población y vivienda 2005, INEGI, México, en www.inegi.gob.mx. [consulta: 1 de abril de 2009].

Instituto Universitario de Desarrollo y Cooperación, 1993, El enfoque del marco lógico. Manual para la planificación de proyectos orientada mediante objetivos, Instituto Universitario de Desarrollo y Cooperación, Universidad Complutense de Madrid, Madrid. 
Ministerio de Asuntos Exteriores, 2001, Metodología de evaluación de la cooperación española, Ministerio de Asuntos Exteriores, Secretaría de Estado para la Cooperación Internacional y para Iberoamérica, Madrid.

March, James y Johan Olsen, 1997, El redescubrimiento de las instituciones. La base organizativa de la política, FCE, Colegio Nacional de Ciencias Políticas y Administración Pública, México.

Martínez Espinoza, Manuel Ignacio, 2009, La participación de los pueblos indígenas en los proyectos de cooperación al desarrollo de la Comisión Europea, Universidad de Salamanca, Tesis doctoral, Salamanca.

Meltsner, Arnold J., 2007, «La factibilidad política y el análisis de políticas», en La hechura de las políticas públicas, editado por Aguilar Villanueva, Luis, Miguel Ángel Porrúa, México, pp. 367-392.

Meny, Ives y Jean Claude Thoening, 1992, Las políticas públicas, Ariel, Barcelona.

Muench, Pablo, 2008, Libro blanco de la selva, Proyecto Desarrollo Social Integrado y Sostenible, Secretaría de Desarrollo Social del Gobierno del Estado de Chiapas, Unión Europea, Tuxtla Gutiérrez.

North, Douglass C., 1995, Instituciones, cambio institucional y desempeño económico, FCE, México.

Osuna, José Luis y Carolina Marquez (editores), 2000, Guía para la evaluación de políticas públicas, Instituto de Desarrollo Regional, Universidad de Sevilla, Sevilla. 
Peters, Guy, 2003, El nuevo institucionalismo: teoría institucional en ciencia política, Gedisa, Barcelona.

Piqueras Infante, Andrés, et al., 2008, Desarrollo y cooperación: un análisis crítico, Tirant lo Blanch, Valencia.

Pressman, Jeffrey y Aaron Wildavsky, 1998, Implementación: cómo grandes expectativas concebidas en Washington se frustran en Oakland, FCE, México.

Prodesis, 2004, Programa operativo global, Unión Europea, Secretaría de Desarrollo Social del Gobierno del Estado de Chiapas, Tuxtla Gutiérrez, en www.prodesis.chiapas.gob.mx/?Plan_Global_y_Planes_Operativos_Anuales [consulta: 10 de enero de 2008].

Quinn Patton, Michael, 1990, Qualitative evaluation and research methods, Sage Publications, Newbury Park.

Rein, Martin y Francine Rabinovitz, 2003, «La implementación: una perspectiva teórica. Entre la intención y la acción», en La implementación de las políticas públicas, editado por Aguilar Villanueva, Luis, Miguel Ángel Porrúa, México, pp. 147-184.

Richardson, Jeremy (editor), 1982, Policy styles in western Europe, Allen and Unwin, London.

Sabatier, Paul y Daniel Mazmanian, 2003, «La implementación de la política pública: un marco de análisis», en La implementación de las políticas públicas, editado por Aguilar Villanueva, Luis, Miguel Ángel Porrúa, México, pp. 323-372. 
Sánchez Ferrer, Leonardo, 2002, «Actores estrategas. La política de educación y el problema de la elección escolar», en Análisis de políticas públicas en España: enfoques y casos, editado por Grau, Mireia y Araceli Mateos, Tirant lo Blanch, Valencia, pp. 221-266.

Sotillo Lorenzo, José Ángel, 2006, Un lugar en el mundo. La política de desarrollo de la Unión Europea, Instituto Universitario de Desarrollo y Cooperación, Universidad Complutense de Madrid, Madrid.

Souto-Otero, Manuel, 2006, «Evaluación de políticas: una revisión crítica de las definiciones, diseños y métodos», en Revista da Escola Galega de Administración Pública. Administración y ciudadanía, n. 1, v. 1, Santiago de Compostela, pp. 173-201.

Tezanos Vázquez, Sergio, 2008, Cooperación para el desarrollo. Asignación geográfica de la ayuda española, Biblioteca Nueva, Madrid.

Universidad Autónoma Metropolitana, 2003-2008, Programa Integral para el Desarrollo Sustentable de la Selva. Evaluación de los ejercicios 2002-2007, Programa de Investigación Interdisciplinario Desarrollo Humano en Chiapas, México.

Uvalle Berrones, Ricardo, 2002, «Los fundamentos institucionales de la gestión pública», en VII Congreso Internacional del CLAD, 8-11 de octubre, Lisboa, en $\quad$ http://unpan1.un.org/intradoc/groups/public/documents/CLAD/clad0043511.pdf [consulta: 6 de agosto de 2008].

Van Meter, Donald y Carl Van Horn, 2003, «El proceso de implementación de las políticas. Un marco conceptual», en La implementación de las políticas públicas, editado por Aguilar Villanueva, Luis, Miguel Ángel Porrúa, México, pp. 97-146. 
Varela de Ugarte, Fernando (dir.), 2007, La calidad de las intervenciones de desarrollo. Fundamentos y herramientas para mejorar el diseño, CIDEAL, Madrid.

Vedung, Evert, 1996, Evaluación de políticas públicas y programas, Instituto Nacional de Servicios Sociales, Ministerio de Trabajo y Asuntos Sociales, Madrid.

\section{NOTAS}

${ }^{1}$ Siguiendo a Ballart, en este artículo se concibe la evaluación dentro de la gestión pública como «la aplicación de métodos de investigación sistemáticos con el objeto de examinar el diseño, la implementación y la utilidad de las políticas y programas públicos» (Ballart 1993: 200). Para reflexiones sobre el término «gestión pública» véase Ballart (1997), Cabrero (2000b), Guerrero (2001) y Uvalle (2002).

${ }^{2}$ Véasen las tipologías que proponen Quinn Paton (1990), Souto-Otero (2006) y Vedung (1996).

${ }^{3}$ Esta clasificación proviene de Osuna y Márquez (2000). Para otros autores como Ballart (1993) y Vedung (1996), los modelos de evaluación constan de dos tipos generales: de resultados y de procesos.

${ }^{4}$ Siguiendo a Iglesia-Caruncho (2005), la cooperación internacional al desarrollo puede entenderse como un subsistema dentro del sistema de relaciones internacionales que tiene el objetivo de colaborar en el crecimiento económico y en el bienestar de los países en desarrollo (Iglesia-Caruncho 2005: 35). En el presente artículo se concibe la cooperación internacional al desarrollo como el «trabajo conjunto de instituciones, organizaciones y actores, tanto del norte como del sur, a través de un conjunto de actuaciones, con el objetivo de lograr el desarrollo de los países pobres» (Sotillo 2006: 105-106).

${ }^{5}$ Las intervenciones al desarrollo se definen como el conjunto de acciones que la cooperación internacional al desarrollo realiza para cumplir con sus objetivos. Las intervenciones pueden tomar forma de proyectos, programas, estrategias o políticas.

${ }^{6}$ La Comisión Europea adoptó la GCP como método de trabajo desde 1993 y ha recomendado a sus Estados miembros usar esta metodología. Asimismo, también el Comité de Ayuda al Desarrollo de la Organización para la Cooperación y el Desarrollo Económico (OCDE) ha sugerido el uso de la GCP.

${ }^{7}$ El EML es una metodología de estructuración de los principales elementos de un proyecto, subrayando los lazos lógicos entre los insumos previstos, las actividades planeadas y los resultados esperados. El EML considera seis fases en el ciclo de intervención (programación, identificación, formulación, ejecución, seguimiento y evaluación) y tres componentes en la matriz de cada intervención: la jerarquía de objetivos, los criterios de evaluación y los factores de desarrollo (IUDC 1993).

${ }^{8}$ Las evaluaciones en la cooperación internacional pueden ser de distintas categorías. Tal vez la tipología más acabada es la de MAE (2001: 116-121), donde las evaluaciones han sido divididas en seis modalidades: evaluaciones de intervenciones puntuales, evaluaciones sectoriales, evaluaciones geográficas, evaluaciones de instrumentos, evaluaciones de programas y evaluaciones temáticas.

${ }^{9}$ Como asienta Arellano, «el enfoque de la política pública ha demostrado que los problemas públicos no son atacables sólo de manera técnica, ya que siempre existirán una multiplicidad de valores para definir el problema. De hecho, el analista de políticas no define el problema, sino que en estricto sentido lo crea» (Arellano 1999: 37).

${ }_{10}$ «Certificado de nacimiento» que no es banal pues se basa en una distinción entre los términos politics y policy; siendo el primero utilizado para referirse a «la competencia política» y el segundo para «un curso definido de acción» (Beltrán 2000: 19). Tal diferencia conceptual está ausente en el mundo hispanoparlante, donde para ambos enfoques se utiliza el término de «política». 
${ }^{11}$ En la literatura especializada es factible encontrar por lo menos siete denominaciones distintas sobre los estudios enfocados en la policy: 1) policy sciences, 2) policy analysis, 3) policy-making study, 4) policy studies, 5) policy style; 6) estudios de implementación de políticas, y 7) evaluación de políticas públicas. En un intento de precisión, Luís Aguilar Villanueva ha establecido que la investigación en políticas públicas se divide en dos grandes rubros: por un lado, el análisis de políticas (policy analysis), orientado normativamente a la búsqueda de la mejor política pública en términos de eficiencia y equidad; y por el otro lado, el estudio de la elaboración de las políticas (policy-making research), orientado positivamente a describir, clasificar y explicar el patrón de decisión y operación con el que procede un sistema político-administrativo dado o un gobierno particular en sus políticas públicas (Aguilar 2007: 42).

12 «En un extremo, se ubica la visión racional estricta del análisis de políticas que puede quizá reconocer la existencia de diversas y poderosas restricciones, pero sin renunciar por ello a la exigencia de la racionalidad máxima posible en la formulación y decisión de la política. En el extremo opuesto, se ubica la visión negociadora, concertadora, pragmática de la política, que utilizará táctica o casuísticamente el análisis, pero que lo considerará una condición insuficiente y en ciertas circunstancias innecesaria para la decisión y desarrollo de la política» (Aguilar 2007: 41).

${ }^{13}$ Como asienta Cabrero (2000), el modelo del policy analysis fue generado y ha sido desarrollado principalmente por y para el espacio anglosajón. Por lo tanto, el autor propugna por entender hasta dónde funciona el modelo en realidades diferentes y hasta dónde algunas realidades pueden hacer uso del método. El autor concluye que «La escuela de las políticas públicas no debe ser un modelo que deba incorporarse acríticamente en diversos contextos en un esfuerzo hasta ahora poco fructífero. Por el contrario, debe ser entendido como un método de análisis por construirse para responder así al cuestionamiento de cuáles son los usos y costumbres en la hechura de políticas públicas en diversas realidades» (Cabrero 2000: 221-222).

${ }^{14}$ Comúnmente, se plantean seis fases de las políticas públicas: identificación, formulación de problemas, formulación y legitimación de alternativas de acción, puesta en marcha de la política, y evaluación.

15 «En los estudios basados en el institucionalismo, el factor clave a la hora de explicar las políticas públicas es el conjunto de normas, valores y reglas de actuación que determinan el comportamiento de los actores políticos. Es decir, no cuenta tanto quiénes son los actores, sus preferencias o sus estrategias, como las normas que se han consolidado en un contexto dado, los valores predominantes en un sistema político o los procedimientos de actuación política y administrativa que se han consolidado en un área política determinada» (Sánchez Ferrer 2002: 223).

${ }^{16}$ Para otros modelos de análisis de la cooperación al desarrollo, revísese: Alonso y Fitzgerald (2003), que se enfoca en la eficacia de la ayuda; Gómez Galán y Sanahuja (2001), que estudia la cooperación desde un análisis del sistema internacional; Iglesia-Caruncho (2005), que observa los impactos socioeconómicos de la cooperación; Piqueras Infante (2008), que compendia análisis críticos sobre la cooperación al desarrollo; y Varela de Ugarte (2007), que se concentra en el diseño de los proyectos.

${ }^{17}$ Un excelente estudio sobre la cooperación al desarrollo que emplea métodos econométricos y, en ese sentido, ejemplifica las herramientas analíticas excluidas en el modelo propuesto, es el de Tezanos Vázquez (2008).

${ }^{18}$ En 2007 se formaron dos microrregiones más: Maravilla Tenejapa se dividió para formar Maravilla Tenejapa y Amatitlán; la que era Río Blanco se dividió para formar Río Blanco y Nuevo Plan de Ayala. De esta manera, el territorio de trabajo del Prodesis aumentó a 18 microrregiones.

${ }^{19}$ Página web del Prodesis:

www.Prodesis.chiapas.gob.mx/?\%BFQu\%E9_es_el_PRODESIS\%3F/Ejes_estrat\%E9gicos_transversales [consulta: 11 de enero de 2008]

${ }^{20}$ La evaluación de la gestión del Prodesis con el modelo de análisis de políticas públicas propuesto implicó un estudio a profundidad realizado entre los años 2007 y 2009. En ese periodo se examinaron los documentos oficiales del proyecto, se realizó observación directa en una parte del territorio donde se implementaron los proyectos (subregión Comitán) y se entrevistó en profundidad a cerca de treinta personas entre funcionarios, ex funcionarios, beneficiarios, académicos y miembros de organizaciones sociales que se habían vinculado 
con el proyecto. Lo que se presenta a continuación es una síntesis de los resultados del estudio. Para profundizar en el caso del estudio del Prodesis véase Martínez Espinoza (2009: 91-216).

${ }^{21}$ De acuerdo a datos correspondientes a 2005 — válidos para el contexto temporal de ejecución del Prodesis-, en la selva Lacandona habitaban 406,820 personas, lo que significa un incremento de 54,199 pobladores respecto al censo del año 2000, donde se contabilizaron 352,621 personas. Los asentamientos de la Lacandona no cuentan ni con infraestructura ni con servicios básicos, y sus habitantes forman parte de una de las zonas más marginadas de México (INEGI, 2005).

${ }^{22}$ Véase De Vos (2002) y Muench (2008).

${ }^{23}$ Uno de los objetivos del PIDSS lo deja muy claro: «reconstruir el tejido social recuperando la legitimidad de la acción gubernamental y el estado de derecho impulsando la participación social, democrática, incluyente y plural» (Muench 2008: 115).

${ }_{24}$ Esta clasificación de la agenda proviene de Elder y Cobb (1996) y Meny y Thoening (1992), para quienes las agendas suelen ser de dos tipos: 1 ) agenda sistémica o coyuntural, que es el conjunto de asuntos aceptados por la sociedad como temas que requieren de la atención de la autoridad pública pero sin ser de su competencia habitual; y 2) agenda institucional o formal, que son aquellas cuestiones que los miembros de una comunidad perciben como merecedores de la habitual atención pública y como asuntos que caen en la habitual jurisdicción legítima de la autoridad gubernamental.

${ }^{25}$ La clasificación del estilo de políticas (policy style) proviene de Richardson (1982).

${ }^{26}$ Meltsner ha definido el espacio de la política (policy space) como el espacio que contiene «aquellos ingredientes políticos que nos permiten comprender una amplia gama de problemas (...). Todo sistema político contiene un número determinado de espacio de políticas que se superponen unos a otros» (Meltsner 2007: 371). Un análisis del policy space facilita el entendimiento del contexto donde se inserta una política.

${ }^{27}$ Esta última desconsideración facilitó argumentos a quienes calificaron al Prodesis como «contrainsurgencia de buena fe» (entrevistas de campo).

${ }^{28}$ En el trabajo de campo se evidenciaron dos obstáculos que afectaron la implementación del Prodesis: por un lado, que ni las instituciones gubernamentales ni los tres niveles de gobierno reconocieron a las microrregiones como espacios de planeación y gestión del desarrollo local. Por otro lado, que las instituciones gubernamentales no fueron corresponsables en los trabajos relativos a sus materias en las microrregiones.

${ }^{29}$ Las competencias cívicas son las expectativas de comportamiento, valores y saberes de los sujetos en función de un modelo deseable de ciudadanía. Se concretan en el conjunto de conocimientos, habilidades y actitudes que preparan a los sujetos para la vida en democracia (IFE 2003: 13).

${ }^{30}$ Para una aplicación más amplia del modelo propuesto, véase Martínez Espinoza (2009).

Fecha de recepción: 14 de octubre de 2010.

Fecha de aceptación: 18 de mayo de 2011. 